\title{
Path Planning for Efficient UAV Coordination in Aerobiological Sampling Missions
}

\author{
Laszlo Techy ${ }^{1}$, Craig A. Woolsey ${ }^{1}$, David G. Schmale III ${ }^{2}$
}

\begin{abstract}
This paper is concerned with the coordinated flight of two autonomous UAVs to be used for aerobiological sampling of biological threat agents above agricultural fields. The periodic sampling task involves two phases: a sampling interval and an initialization interval. During the sampling interval, both vehicles must employ their aerobiological sampling devices and follow a precise ground track in the presence of sustained winds. During the initialization interval, the vehicles move to their respective initial states to begin the next sampling interval. To maximize the volume of air sampled by the UAVs during an individual sampling mission, the initialization interval must be as short as possible. The paper provides a simple, geometric method for generating candidate timeoptimal paths in steady winds, based on Dubins' well-known results for minimum time paths of bounded curvature. The approach is used to generate paths for both UAVs, which must coordinate their motion along their respective paths in order to avoid collision. The described methods were tested during an aerobiological sampling experiment focusing on the plant pathogen Phytophthora infestans.
\end{abstract}

\section{INTRODUCTION}

This paper focuses on the use of small UAVs for aerobiological sampling, an application which enables plant pathologists to detect, monitor, and forecast the spread of high risk plant pathogens from tens to hundreds of meters above agricultural fields [12]. Recent research, in which small UAVs were used to collect samples of airborne biological particles, such as pollen or mold spores, in the lower atmosphere gives conclusive evidence that common plant pathogens can be transported over much greater distances than had previously been assumed [6]. UAVs used for aerobiological sampling are fitted with spore-sampling devices, consisting of circular petri plates that can be opened and closed like a clam shell while the UAV is in flight; see Figure 1. The petri plates are immediately removed from the airplanes after the flight and transferred to the laboratory for later cultivation.

The use of small UAVs for aerobiological sampling suggests a number of important and fascinating control challenges, including fast, adaptive control to reject large disturbances [14], multi-UAV coordination, and precision navigation in significant winds. One compelling problem is the "control volume sampling" task. In this scenario, two UAVs fly closed paths at a common altitude above an agricultural field suspected of hosting a high-risk plant

${ }^{1}$ Department of Aerospace \& Ocean Engineering, ${ }^{2}$ Department of Plant Pathology, Physiology, and Weed Science, Virginia Tech, Blacksburg, VA 24061 techy, cwoolsey, dschmale\}evt.edu

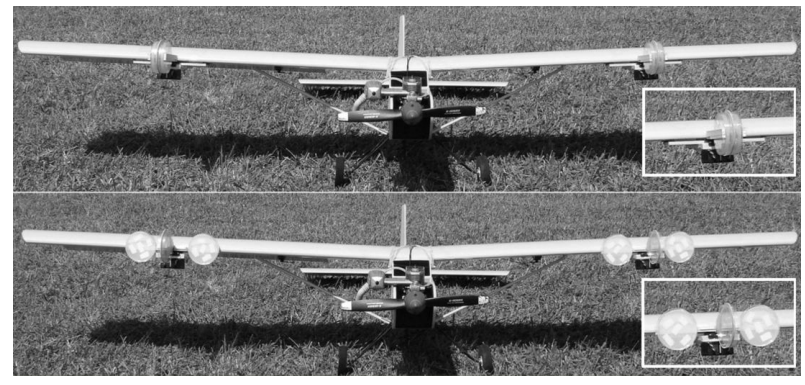

Fig. 1. A UAV with sample collection plates closed (top) and open (bottom) [6]. In the control volume sampling application, the plates are opened only during the sampling interval.

pathogen. The UAVs modulate their sampling activity so that one UAV samples only when it is upwind of the field and the other samples only when it is downwind; see Figure 4. Because the two paths overlap, the UAVs are programmed to maximize their "phase difference" to minimize the chance of collision. The resulting samples allow researchers to more accurately characterize pathogen release within the sampled region. When the UAVs are not sampling, they are "re-initializing" their position and course angle to sample once again, preferably as quickly as possible in order to minimize the time and fuel that is spent with the sample plates closed. If each aircraft had multiple, independently actuated sampling devices, both aircraft could simply fly circular paths, opening one sampling device while flying upwind of the field and the other while flying downwind. The proposed approach is effective when it is not feasible to alternate between sampling devices, because they are not independently actuated, for example, or because the vehicle can only carry a single device.

There is a considerable volume of literature on control of planar formations. In [3], control laws were derived to ensure asymptotic stability of relative equilibria for planar formations. The same model was studied in [13], which considered formation control in the context of nonlinear oscillator synchronization. The model was also used in [5], where the objective involved tracking a moving target. Small UAVs fly at low speeds and are invariably subject to significant winds, particularly in the aerobiological sampling task, where ambient wind drives particle transport. If unaccounted for, winds can substantially degrade the performance of a UAV guidance system [15]. Recent efforts in coordinated 
control of ocean gliders have addressed the problem of coordination of multiple autonomous agents along general convex curves [9]. Coordination in the presence of winds is addressed in [8].

This paper suggests an approach to coordinated control volume sampling for aerobiological research using two UAVs. A key contribution is the development of a simple, geometric path planning procedure for time-optimal flight in a steady wind. The paper is organized as follows. In Section II, we set up the problem of computing a minimum time path, given an initial position and heading and a final position and heading in the presence of a steady wind. Section III describes a procedure for constructing a candidate minimum time path. In Section IV, we propose a phase synchronization method, and in Section $\mathrm{V}$ we discuss the experimental results of both the path planning algorithm and the synchronization method. Section VI provides conclusions and describes our ongoing work on this problem.

\section{Problem Statement}

To address the objectives outlined in the introduction, we separate each UAV's periodic flight path into two periodic portions: a sampling interval and an initialization interval.

Sampling Interval. In the sampling interval, each UAV opens its air samplers and regulates its course angle in order to track a semicircular inertial path, denoted by "U" for the upwind sampler or "D" for downwind sampler.

Initialization Interval. During the initialization interval, each UAV closes its air samplers and prepares to begin the next sample interval. Since the UAVs are wasting time and fuel during this interval, they should "queue up" to start the next sample arc as quickly as possible. Thus, assuming flight at constant airspeed, we seek, for each UAV, the minimum time path from the end of one sample interval to the beginning of the next. Noting that the wind speed and direction may drift over the course of a sampling mission, we seek a path optimization method that is simple enough to solve repeatedly and quickly.

Consider the dynamic system described by the equations

$$
\begin{aligned}
\dot{x}_{N}(t) & =V_{a} \cos \psi(t)+V_{x} \\
\dot{y}_{E}(t) & =V_{a} \sin \psi(t)+V_{y} \\
\dot{\psi}(t) & =u(t),
\end{aligned}
$$

where the components $V_{x}$ and $V_{y}$ of the ambient wind are assumed to be constant. The coordinates $x_{N}(t)$ and $y_{E}(t)$ describe the vehicle's position in an inertial frame while $\psi(t)$ describes its heading relative to the $x_{N}$-axis. $V_{a}$ is the airspeed and $u(t)$ is the turn rate, which we take as a control input.

Suppose the initial and desired terminal conditions are

$$
\begin{aligned}
& x_{N}(0)=x_{N_{0}}, \quad y_{E}(0)=y_{E_{0}}, \quad \psi(0)=\psi_{0}, \\
& x_{N}(T)=x_{N_{f}}, \quad y_{E}(T)=y_{E f}, \quad \psi(T)=\psi_{f} .
\end{aligned}
$$

The objective is to find an extremal control $u^{*}(t)$ such that the UAV, starting from its initial state, arrives at the desired

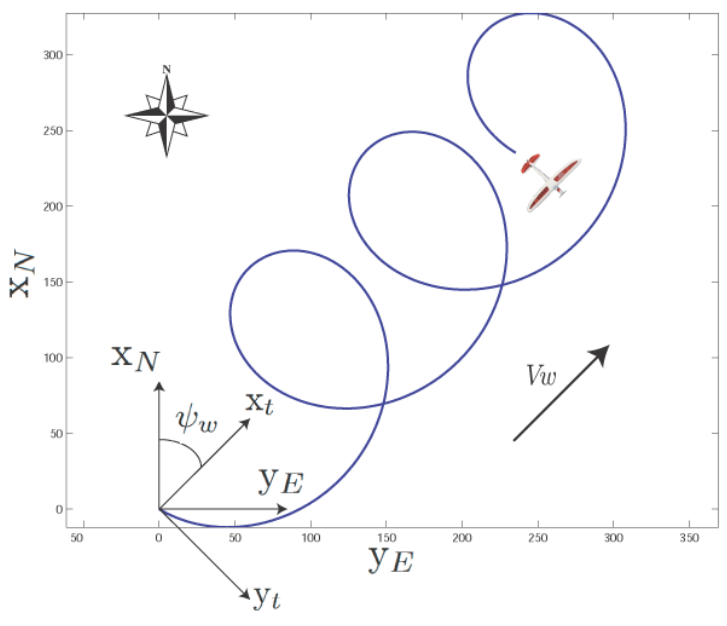

Fig. 2. Trochoidal path and trochoidal frame.

final state in minimum time. That is, the objective is to find $u^{*}(t)$, such that the cost function

$$
J=\int_{0}^{T} d t=T
$$

is minimized subject to the kinematic equations (1) and the symmetric control limits

$$
-u_{\max } \leq u(t) \leq u_{\max } .
$$

Finding minimum length paths of bounded curvature was studied by Dubins [2] and was more recently adopted as a means of generating minimum-time paths for constant-speed mobile robots with bounded turn rates, leading to the phrase "Dubins' car." (See [1], for example.) One attempt to extend Dubins' results to UAVs in winds is described in [7], where the problem is transformed to a moving reference frame in which the final position becomes a "virtual moving target" whose speed is equal and opposite to the wind. Path planning then reduces to a numerical root-finding problem involving iterative solution of the Dubins problem.

Section III describes an alternative algorithm that uses a simple geometric argument to generate a subset of the extremal paths. The result relies on the observation that circular (constant turn rate) UAV paths in the air-relative frame correspond to trochoidal paths in the inertial frame [11]. Following [11], we define a trochoidal frame determined by the wind direction, as shown in Figure 2. If $\chi_{w}$ denotes the wind direction (by convention, the direction from which the wind approaches), then $\psi_{w}=\chi_{w} \pm \pi$ is the direction of the ambient air's motion. The trochoidal frame is then defined such that its $x$-axis is oriented downwind, the $z$ axis is into the image, and the $y$-axis completes the righthanded reference frame. Then the trochoidal coordinates can be found from the inertial coordinates by

$$
\left(\begin{array}{l}
x_{t}(t) \\
y_{t}(t)
\end{array}\right)=\left(\begin{array}{cc}
\cos \psi_{w} & \sin \psi_{w} \\
-\sin \psi_{w} & \cos \psi_{w}
\end{array}\right)\left(\begin{array}{l}
x_{N}(t) \\
y_{E}(t)
\end{array}\right) .
$$


The kinematic equations expressed in the trochoidal frame are

$$
\begin{aligned}
& \dot{x}_{t}(t)=V_{a} \cos \left(\psi(t)-\psi_{w}\right)+V_{w} \\
& \dot{y}_{t}(t)=V_{a} \sin \left(\psi(t)-\psi_{w}\right) \\
& \dot{\psi}(t)=u(t)
\end{aligned}
$$

where $V_{w}=\sqrt{V_{x}^{2}+V_{y}^{2}}$. We assume that $V_{w}<V_{a}$, to ensure that feasible solutions exist. In the case of a turn at constant maximum rate $\omega=\left|\dot{\psi}_{\max }\right|$, the equations can be re-written as

$$
\begin{aligned}
& \dot{x}_{t}(t)=V_{a} \cos \left(\delta \omega t+\phi_{t}\right)+V_{w} \\
& \dot{y}_{t}(t)=V_{a} \sin \left(\delta \omega t+\phi_{t}\right),
\end{aligned}
$$

where $\phi_{t}=\psi(0)-\psi_{w}$ and $\delta \in\{-1,1\}$ describes the direction of the turn. The position of a point on the trochoidal path can then be written as

$$
\begin{aligned}
& x_{t}(t)=\frac{V_{a}}{\delta \omega} \sin \left(\delta \omega t+\phi_{t}\right)+V_{w} t+x_{t_{0}} \\
& y_{t}(t)=-\frac{V_{a}}{\delta \omega} \cos \left(\delta \omega t+\phi_{t}\right)+y_{t_{0}} .
\end{aligned}
$$

The trochoidal path defined here is essential in developing the path planning algorithm described in the next section.

\section{PATH Optimization}

This section focuses on the initialization phase, where each UAV must attain the prescribed initial position and course angle to begin the next sample interval. Following [1], we begin with equations (1) and initial conditions (2). Without loss of generality, assume that $V_{y}=0$. The Hamiltonian for the time-optimal control problem is

$$
H=1+\lambda_{1}\left(V_{a} \cos \psi(t)+V_{w}\right)+\lambda_{2} V_{a} \sin \psi(t)+\lambda_{3} u,
$$

where $u \in \mathscr{U}$, with $\mathscr{U}$ being the set of admissible controls. The co-state equations are

$$
\begin{aligned}
& \dot{\lambda}_{1}=0 \\
& \dot{\lambda}_{2}=0 \\
& \dot{\lambda}_{3}=\lambda_{1} V_{a} \sin \psi(t)-\lambda_{2} V_{a} \cos \psi(t)
\end{aligned}
$$

which implies that $\lambda_{1}$ and $\lambda_{2}$ are constant. The minimum principle states that, along an extremal trajectory,

$$
H\left(x^{*}, u^{*}, \lambda^{*}, t\right) \leq H\left(x^{*}, u, \lambda^{*}, t\right)
$$

for all $u \in \mathscr{U}$ and $0 \leq t \leq T$. First assume that $\lambda_{3} \neq$ 0 . To satisfy Pontryagin's minimum principle, one needs $u=-\operatorname{sign}\left(\lambda_{3}\right) u_{\max }$, which is a maximum rate turn to the left or right, a "maximum effort" or "bang-bang" control. Next consider the case where $\lambda_{3} \equiv 0$. Since by Pontryagin's minimum principle $\lambda$ must be nonzero, and since $\lambda_{1}$ and $\lambda_{2}$ are constant,

$$
\lambda \|\left(\begin{array}{c}
\cos \psi(t) \\
\sin \psi(t) \\
0
\end{array}\right)
$$

in order to satisfy the adjoint equations. It follows that $\psi(t)$ must be constant, which corresponds to a straight path. Thus, the time-optimal path consists of turns at maximum rate and straight segments. Extremal trajectories therefore comprise trochoidal and straight segments. As argued in [7] there exist four distinct, candidate optimal paths (when the initial and final points are sufficiently far apart, such as the case we are considering), each of which involves a turn at maximum rate, a straight line and a final turn at maximum rate, equivalently two trochoids connected by a straight segment.

Given the initial and final conditions (2) and (3), we define two trochoids $\left(x_{t_{1}}(t), y_{t_{1}}(t)\right)^{T}$ and $\left(x_{t_{2}}(t), y_{t_{2}}(t)\right)^{T}$ as in (6) and (7), such that the first trochoid satisfies the initial conditions (2) at $t=0$, and the second trochoid satisfies the final conditions (3) at $t=t_{2 \pi}=\frac{2 \pi}{\omega}$, the time required for the air-relative velocity vector to describe a full circle at the maximum turn rate. We let $\delta_{i}$ denote the sense of the turn for $i \in\{1,2\}$.

$$
\begin{aligned}
x_{t_{1}}(t) & =\frac{V_{a}}{\delta_{1} \omega} \sin \left(\delta_{1} \omega t+\phi_{t_{1}}\right)+V_{w} t+x_{t_{10}} \\
y_{t_{1}}(t) & =\frac{-V_{a}}{\delta_{1} \omega} \cos \left(\delta_{1} \omega t+\phi_{t_{1}}\right)+y_{t_{10}} \\
x_{t_{2}}(t) & =\frac{V_{a}}{\delta_{2} \omega} \sin \left(\delta_{2} \omega t+\phi_{t_{2}}\right)+V_{w} t+x_{t_{20}} \\
y_{t_{2}}(t) & =\frac{-V_{a}}{\delta_{2} \omega} \cos \left(\delta_{2} \omega t+\phi_{t_{2}}\right)+y_{t_{20}}
\end{aligned}
$$

Remark 3.1: There is a slight abuse of notation here, in using the same path parameter $t$ for both curves.

Let us denote the point at which the extremal path leaves the first trochoidal segment as point $A$, and the point where it reaches the second trochoidal segment as point $B$ (Figure 3 ). Define $t_{A}$ and $t_{B}$, relative to the two trochoidal segments, such that

$$
\left(\begin{array}{l}
x_{A} \\
y_{A}
\end{array}\right)=\left(\begin{array}{l}
x_{t_{1}}\left(t_{A}\right) \\
y_{t_{1}}\left(t_{A}\right)
\end{array}\right) \text { and }\left(\begin{array}{l}
x_{B} \\
y_{B}
\end{array}\right)=\left(\begin{array}{l}
x_{t_{2}}\left(t_{B}\right) \\
y_{t_{2}}\left(t_{B}\right)
\end{array}\right)
$$

In order for a given connecting line segment to belong to an extremal path, the following conditions must be satisfied

- The velocities at point $A$ and point $B$ must be equal:

$$
\left(\dot{x}_{t_{1}}\left(t_{A}\right), \quad \dot{y}_{t_{1}}\left(t_{A}\right)\right)^{T}=\left(\dot{x}_{t_{2}}\left(t_{B}\right), \quad \dot{y}_{t_{2}}\left(t_{B}\right)\right)^{T} .
$$

- The line segment joining the points $A$ and $B$ must be tangent with the velocity vectors at both points:

$$
\begin{aligned}
\tan (\alpha) & =\frac{x_{t_{2}}\left(t_{B}\right)-x_{t_{1}}\left(t_{A}\right)}{y_{t_{2}}\left(t_{B}\right)-y_{t_{1}}\left(t_{A}\right)} \\
& =\frac{\dot{x}_{t_{2}}\left(t_{B}\right)}{\dot{y}_{t_{2}}\left(t_{B}\right)}=\frac{\dot{x}_{t_{1}}\left(t_{A}\right)}{\dot{y}_{t_{1}}\left(t_{A}\right)} .
\end{aligned}
$$

- The path parameters must satisfy

$$
t_{A}, t_{B} \in\left[0, t_{2 \pi}\right]
$$


Using equations (4)-(5), condition (12) can be written as

$$
\delta_{1} \omega t_{A}+\phi_{t_{1}}=\delta_{2} \omega t_{B}+\phi_{t_{2}}
$$

Expressing $t_{A}$ as a function of $t_{B}$, and using equations (8)(11) and (13)-(14), we find

$$
E \cos \left(\delta_{2} \omega t_{B}+\phi_{t_{2}}\right)+F \sin \left(\delta_{2} \omega t_{B}+\phi_{t_{2}}\right)=G
$$

where

$$
\begin{aligned}
E & =V_{a}\left(V_{w} \frac{\delta_{1}-\delta_{2}}{\delta_{1} \delta_{2} \omega}-\left(y_{t_{20}}-y_{t_{10}}\right)\right) \\
F & =\left(\left(x_{t_{20}}-x_{t_{10}}\right)+V_{w} t_{B}\left(1-\frac{\delta_{1}}{\delta_{2}}\right)+\frac{\phi_{t_{1}}-\phi_{t_{2}}}{\delta_{1} \omega}\right) \\
G & =V_{w}\left(y_{t_{20}}-y_{t_{10}}\right)-\frac{V_{a}^{2}\left(\delta_{1}-\delta_{2}\right)}{\delta_{1} \delta_{2} \omega} .
\end{aligned}
$$

Equation (17) has the only unknown: $t_{B}$. Having found $t_{B}$, one may solve for $t_{A}$ using (16). Because (17) is transcendental, $t_{B}$ must be found numerically, in general. However, the problem is significantly simplified if one assumes that

$$
\operatorname{sign}\left(\delta_{1}\right)=\operatorname{sign}\left(\delta_{2}\right)
$$

that is, that the two trochoids have the same sense. In this case

$$
E=V_{a}, \quad F=-V_{a} \tan (\alpha), \quad G=-V_{w},
$$

and

$$
\tan (\alpha)=\frac{V_{w} \frac{\left(\phi_{t_{1}}-\phi_{t_{2}}\right)}{\left(\delta_{1} \omega\right)}+\left(x_{t_{20}}-x_{t_{10}}\right)}{\left(y_{t_{20}}-y_{t_{10}}\right)} .
$$

Under this assumption, equation (17) has two solutions for $t_{B}$, one of them can be discarded by enforcing the third constraint (15).

Proposition 3.2: Define the path $\gamma(t), t \in[0, T]$, such that

$$
\begin{array}{ll}
\gamma(t)=\left(\begin{array}{l}
x_{t_{1}}(t) \\
y_{t_{1}}(t)
\end{array}\right) & t \in\left[0, t_{A}\right] \\
\gamma(t)=\left(\begin{array}{l}
x_{t_{1}}\left(t_{A}\right)+\dot{x}_{t_{1}}\left(t_{A}\right)\left(t-t_{A}\right) \\
y_{t_{1}}\left(t_{A}\right)+\dot{y}_{t_{1}}\left(t_{A}\right)\left(t-t_{A}\right)
\end{array}\right) & t \in\left[t_{A}, t_{\beta}\right] \\
\gamma(t)=\left(\begin{array}{l}
x_{t_{2}}\left(t-t_{\beta}+t_{B}\right) \\
y_{t_{2}}\left(t-t_{\beta}+t_{B}\right)
\end{array}\right) & t \in\left[t_{\beta}, T\right],
\end{array}
$$

where

$$
\begin{aligned}
t_{\beta}= & t_{A}+ \\
& \frac{\sqrt{\left(x_{t_{2}}\left(t_{B}\right)-x_{t_{1}}\left(t_{A}\right)\right)^{2}+\left(y_{t_{2}}\left(t_{B}\right)-y_{t_{1}}\left(t_{A}\right)\right)^{2}}}{\sqrt{\dot{x}_{t_{2}}\left(t_{B}\right)^{2}+\dot{y}_{t_{2}}\left(t_{B}\right)^{2}}} \\
T= & t_{\beta}+\left(t_{2 \pi}-t_{B}\right),
\end{aligned}
$$

and $t_{A}$ and $t_{B}$ are found by solving equations (16) and (17). The path coordinate functions and their rates are given by equations (8)-(11) and their time derivatives. The path $\gamma(t)$ satisfies the necessary conditions for time-optimality.

Remark 3.3: The final path that is selected by the algorithm satisfies the necessary conditions for optimality, however one may not conclude optimality, in general, as there are two additional extremal paths to consider - those for which the two trochoidal segments have opposite sense. Considering the specific aerobiological sampling problem

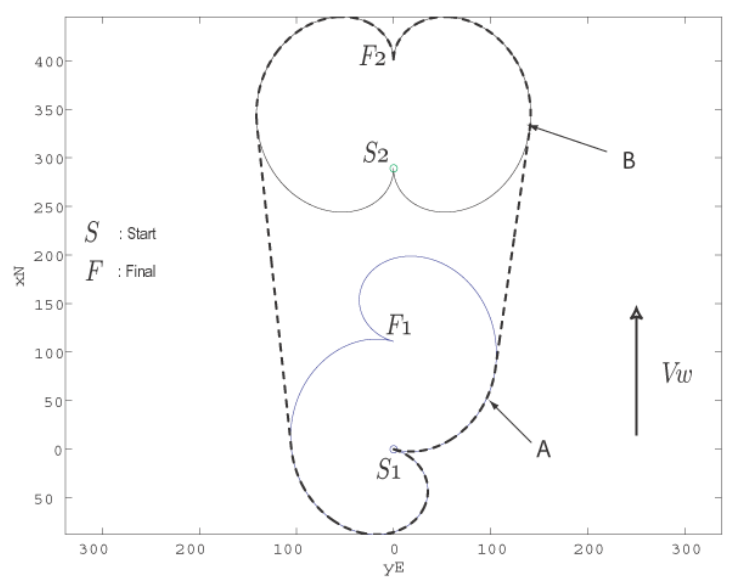

Fig. 3. The dashed line shows candidate extremal paths from initial point and heading to final point and heading. The solid lines are the trochoidal segments at the initial and final states for one full revolution of the airrelative velocity vector.

addressed in this paper, however, one may immediately conclude that this procedure produces the minimum time path. We conjecture that for any closed curve that bounds a convex region in the plane, the time optimal trajectory connecting any two points on the curve has to be of type $B S B$, where $B$ corresponds to a maximum bank angle turn in the same sense as the curve is oriented and $S$ corresponds to a straight segment (a singular arc).

Figure 4 illustrates the concept. In nominal conditions, each UAV's path during the sampling interval is a semicircle. Each UAV's path during the initialization interval is the time-optimal segment connecting the endpoints of the semi-circle.

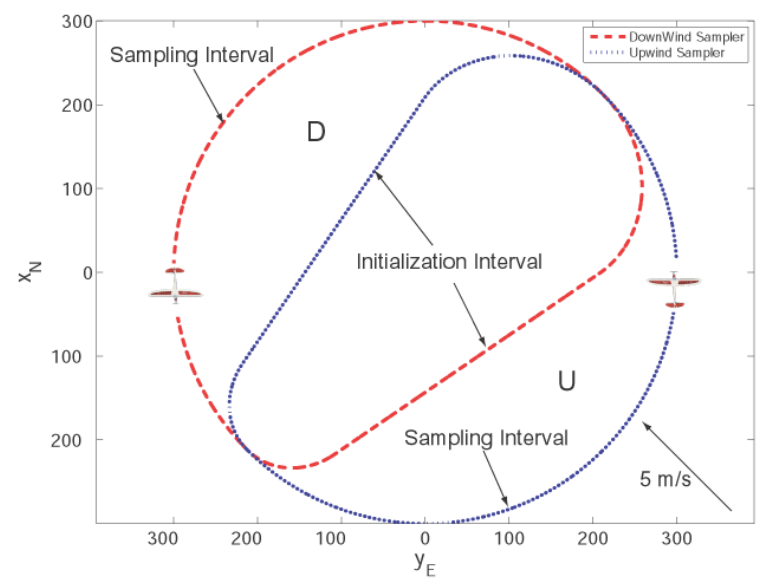

Fig. 4. Two UAVs flying in a time-optimal sampling mission. In the control volume sampling application, UAV1 (UAV2) opens its sample plates only during the upwind (downwind) path segment. 


\section{SYNCHRONIZATION}

In the previous section, we described how to find timeoptimal trajectories with prescribed initial and final positions and headings in the presence of constant wind. In this section, we describe an approach to synchronize the motion of two UAVs so that they are properly phased to avoid collision. We assume the presence of an autopilot that is able to track the desired path at a specified speed. The tracker algorithm can implement waypoint guidance or a more sophisticated guidance strategy, such as the curvilinear path following strategy described in [10]. The method we employ achieves synchronization by changing the speed of the vehicles by a small amount relative to the nominal flight speed. As a first step we present a simple control law for coordination on a circle. Let us denote the "phase angle" of the vehicles by

$$
\theta_{k}(t)=\arctan \left(\frac{y_{E_{k}}(t)}{x_{N_{k}}(t)}\right), \quad k=1,2,
$$

and use a simple particle kinematic model for motion around a circle of radius $R$ (as tracked by the autopilot):

$$
\dot{\theta}_{k}(t)=\omega_{k}(t)=\frac{v_{k}(t)}{R} .
$$

Define

$$
\delta \theta(t)=\theta_{2}(t)-\theta_{1}(t)-\theta^{*}, \quad \delta \theta(t) \in[-\pi, \pi]
$$

as the phase error, where $\theta^{*} \in[0, \pi]$ is the desired phase advantage of $\mathrm{UAV}_{2}$ to $\mathrm{UAV}_{1}$. We assume that

$$
v_{k}(t)=V_{a}+u_{k}(t), \quad k=1,2,
$$

where $V_{a}$ is the desired average airspeed and $u_{k}$ is a control signal. Select the control signal as

$$
u_{k}(t)=K(-1)^{k} \sin (\delta \theta(t))
$$

where $K>0$. Then the phase error dynamics takes the form

$$
\dot{\delta \theta}(t)=-\frac{2 K}{R} \sin (\delta \theta(t))
$$

Lemma 4.1: The origin of the system (20), corresponding to the desired phase arrangement is almost globally asymptotically stable.

The proof is a simple application of Lyapunov's direct method, with the Lyapunov function $V(t)=\frac{1}{2} \delta \theta(t)^{2}$, and LaSalle's invariance principle [4].

In order to eliminate errors due to slight differences in the calibration of the Pitot tube readings, an integral channel can be added as follows. Define

$$
e_{k}(t)=(-1)^{k} \sin (\delta \theta(t))
$$

Take the control signal for the $k^{\text {th }}$ vehicle to be

$$
u_{k}(t)=K_{p} e_{k}(t)+K_{i} \int_{0}^{t} e_{k}(t) d t, \quad k=1,2 .
$$

This control law essentially strives to enforce the condition that the line segment connecting the two UAVs always passes through the geometric center of the circles. The proof has been given for circles, but the method can be used for more general curves, like the one generated by the path planning algorithm of Section III.

\section{EXPERIMENTAL RESUlts}

A series of field experiments were conducted at Virginia Tech's Kentland Farm in July and August, 2008. Two potato fields, covering approximately 3 acres, were inoculated with a domestic strain of Phytophthora infestans, causal agent of late blight disease of potato and tomato. P. infestans is a fungus-like organism that produces infectious propagules called sporangia that may be transported through the atmosphere to healthy plants. UAV flights were coordinated during peak sporangia release (approximately 8 am to $1 \mathrm{pm}$ daily) from the inoculated potato fields.

The path planning algorithm along with the synchronization method was implemented and flight tested at the field trials. In Figure 5 telemetry plots for two UAVs following a 16 minute sampling flight are displayed. The sampling flight was performed on August 19, 2008, 11:10 am (EST) above inoculated potato fields at Kentland Farm. Wind speed and direction were estimated as $4 \mathrm{~m} / \mathrm{s}$ from the west based on data recorded from an on-site weather station during a 20minute interval immediately prior to the sampling mission. The UAVs synchronized their flight using the method described in Section IV, and opened and closed their sampling devices autonomously based on their relative position on the path. The gains for the velocity control algorithm (21) were chosen as $K_{p}=4$ and $K_{i}=\frac{K_{p}}{80}$. Figure 6 shows the phase error time history to illustrate the effectiveness of the synchronization algorithm. The average phase error was $\overline{\delta \theta}=-5^{\circ}$ with standard deviation of $15^{\circ}$.

The average length of the sampling interval was 53 seconds and the average length of the initialization interval was 37 seconds. The total mission lasted 16 minutes which corresponds to 10 periods. The time saved by following timeoptimal initialization paths rather than continuing on a circular trajectory thus resulted in roughly a $16 \%$ improvement in sampling efficiency.

\section{Conclusions}

This paper describes an analytical method for generating candidate minimum time paths for UAVs flying in constant winds, in the context of a control volume sampling scenario for aerobiological data collection. The assumption of constant winds makes it possible to describe the constant airspeed UAV trajectories in terms of line segments and trochoids. This enables a geometric characterization of the extremal paths, making the method suitable for real-time implementation. 


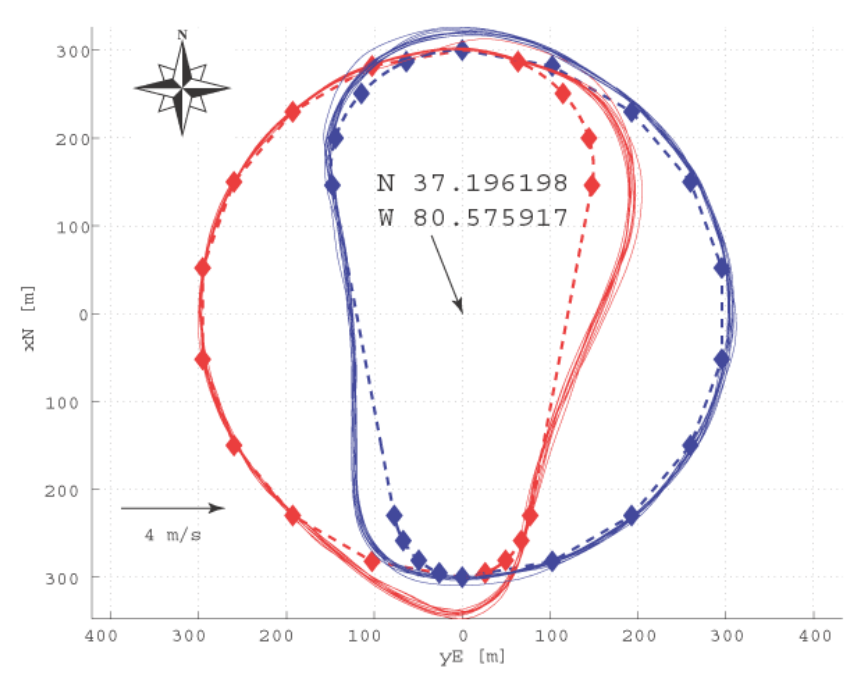

Fig. 5. Experimental results of the path planning algorithm. $\mathrm{UAV}_{1}$ (blue) sampling only downwind from the source, $\mathrm{UAV}_{2}$ (red) sampling only upwind from the source. Diamonds indicate GPS waypoints tracked by the onboard autopilot.

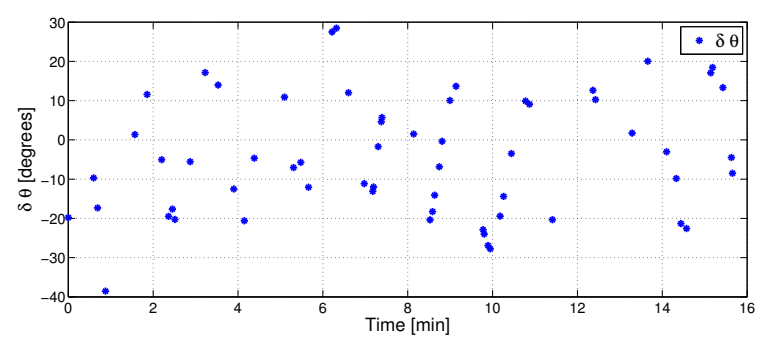

Fig. 6. The effectiveness of the synchronization algorithm as indicated by the phase error (18) between the two UAVs during an aerobiological sampling mission of $P$. infestans. The average phase error was $5^{\circ}$.

Combining the path planning approach with a suitable coordination strategy yields an effective procedure for high capacity aerobiological control volume sampling. The methods developed here were implemented and tested in 2008 field experiments that focused on detecting and monitoring the movement of a high risk plant pathogen, P. infestans, the causal agent of late blight of potato and tomato. The project's larger goals are to enhance the protection and safety of the nation's agriculture and food supply and to develop new strategies to anticipate, respond to, and prevent damage caused by high risk plant pathogens.

A number of outstanding issues remain to be addressed. There is an ongoing effort to implement the nonlinear oscillator approach for maintaining a balanced formation throughout the periodic orbits [9], where the paths can have zero curvature segments. The paper's more basic contribution, a procedure for generating candidate time-optimal paths for UAVs flying in steady winds, can be further strengthened by investigating the two remaining candidate paths, for which no analytical expressions were found. Although the optimal path planning procedure presented here was developed for a specific application, we anticipate that it may have broader use in optimal path generation for unmanned aerial or marine vehicles in the presence of steady winds or currents. The results are immediately applicable, for example, to the problem of optimal path planning for underwater gliders in ocean currents.

\section{ACKNOWLEDGEMENTS}

The project was supported in part by the National Research Initiative of the USDA Cooperative State Research, Education and Extension Service, grant number 2008-5560518673. The authors gratefully acknowledge the input of the anonymous reviewers. The first author would like to acknowledge Justin Murtha for his help with the flight tests.

\section{REFERENCES}

[1] J. D. Boissonat, A. Cerezo, and J. Leblond. Shortest paths of bounded curvature in the plane. In Proc. of the IEEE International Conference on Robotics and Automation, Nice, France, May 1992.

[2] L. E. Dubins. On curves of minimal length with a constraint on average curvature, and with prescribed initial and terminal positions and tangents. American Journal of Mathematics, 79, 1957.

[3] E. W. Justh and P. S. Krishnaprasad. Equilibria and steering laws for planar formations. Systems and Control Letters, 52:25 - 38, 2004.

[4] H. K. Khalil. Nonlinear Systems. Prentice Hall, Upper Saddle River, NJ, third edition, 2002.

[5] D. J. Klein and K. A. Morgansen. Controlled collective motion for trajectory tracking. In Proc. of American Control Conf., Minneapolis, MN, June 2006.

[6] S. L. Maldonado-Ramirez, D. G. Schmale III, E. J. Shields, and G. C. Bergstrom. The relative abundance of viable spores of Gibberella $z e a e$ in the planetary boundary layer suggests the role of long-distance transport in regional epidemics of Fusarium head blight. Agricultural and Forest Meteorology, 132:20-27, 2005.

[7] T. G. McGee, S. Spry, and J. K. Hedrick. Optimal path planning in a constant wind with a bounded turning rate. In AIAA Guidance Navigation and Control Conference and Exhibit, San Francisco, CA, August 2005.

[8] D. A. Paley. Cooperative control of an autonomous sampling network in an external flow field. In Proceedings of the 47th IEEE Conference on Decision and Control, Cancun, Mexico, December 2008.

[9] D. A. Paley, N. E. Leonard, and R. Sepulchre. Stabilization of symmetric formations to motion around convex loops. Systems and Control Letters, 57(3):209 - 215, March 2008.

[10] R. T. Rysdyk. Unmanned aerial vehicle path following and target observation in wind. Journal of Guidance Control and Dynamics, 29(5): 1092 - 1100, 2006.

[11] R. T. Rysdyk. Course and heading changes in significant wind. Journal of Guidance Control and Dynamics, 30(4):1168 - 1171, 2007.

[12] D. G. Schmale, B. R. Dingus, and C. Reinholtz. Development and application of an autonomous unmanned aerial vehicle for precise aerobiological sampling above agricultural fields. Journal of Field Robotics, 25(3): 133 - 147, 2008.

[13] R. Sepulchre, D. A. Paley, and N. E. Leonard. Stabilization of planar collective motion: All-to-all communication. IEEE Transactions on Automatic Control, 52(5):811 - 824, 2007.

[14] J. Wang, V. V. Patel, C. A. Woolsey, N. Hovakimyan, and D. G. Schmale III. L1 adaptive control of a uav for aerobiological sampling. In Proceedings of the 2007 American Control Conference, New York, NY, July 2007.

[15] R. A. Wise and R. T. Rysdyk. Uav coordination for autonoumous traget tracking. In AIAA Guidance Navigation and Control Conference and Exhibit, Keystone, CO, August 2006. 